\title{
APPENDIX ONE
}

\section{HOUSEHOLDS INFECTED WITH \\ PLAGUE, 1542-46}

\begin{tabular}{|c|c|c|}
\hline Person / Household & Details & Date \\
\hline Villager in Champel & Dead of plague & 23 October 1542 \\
\hline Jean de Cortelles & Dead of plague & 29 October 1542 \\
\hline Rose Inn & Infected, to be cleaned & 3 November 1542 \\
\hline Claude Malbuisson & One woman infected & 3 November 1542 \\
\hline Oddet Chenellet & One woman infected & 3 November 1542 \\
\hline Magestray house & Infected & 13 November 1542 \\
\hline Heustace Vincent, herald & Infected & 25 January 1543 \\
\hline Wife of Tyvent Furjod & Suspected of plague & 27 April 1543 \\
\hline François Boulat & Plague-carrying & 27 April 1543 \\
\hline Croset, the butcher & House infected & 17 May 1543 \\
\hline Michel Varro & One girl infected & 21 May 1543 \\
\hline Richard Garnesse & Possibly plague-carrying & 21 May 1543 \\
\hline Pierre Blanchet, minister & Dead of plague & 1 June 1543 \\
\hline Jean Pernet (or Rojon), barber & Dead of plague & 2 June 1543 \\
\hline Joly Cler & House infected & 18 June 1543 \\
\hline Pierre de Veyrer & Infected & 26 June 1543 \\
\hline Jean Bertier & Infected & 15 July 1543 \\
\hline Estienne de la Maisonneuve & Infected & 19 July 1543 \\
\hline Buguyet & Husband and wife dead of plague & 3 September 1543 \\
\hline Guillaume Marchant & $\begin{array}{l}\text { Dead of plague, estate left to Plague } \\
\text { Hospital }\end{array}$ & 23 October 1543 \\
\hline Antoine Bronges & Entire family dead of plague & 23 October 1543 \\
\hline Canestran, labourer & Wife dead of plague & 26 October 1543 \\
\hline Tyvent Laurent & Infected & 11 December 1543 \\
\hline Unnamed debtor & Dead of plague, goods sold & 21 December 1543 \\
\hline Jean Collomb & Girl dead of plague & 30 August 1544 \\
\hline Jean Fiollet & Possible plague illness & 6 October 1544 \\
\hline François Bel & Wife dead of plague & 6 October 1544 \\
\hline Pierre Compagnion & Dead of plague & 23 October 1544 \\
\hline George Malliet & House infected & 15 September 1545 \\
\hline Christophle Suijod & Dead of plague, estate left to city & 6 November 1544 \\
\hline
\end{tabular}


APPENDIX ONE

\begin{tabular}{lll}
\hline Person /Household & Details & Date \\
\hline Pierre Roph (or Taborin) & Quarantine violations & 4 December 1544 \\
Foreigner at Madeleine & Unknown illness, banished & 13 January 1545 \\
François Charnet & Fathers house infected & 27 January 1545 \\
Claudaz Mossier (or Peytavin) & Infected with plague & 12 March 1545 \\
Two houses in St-Gervaix & Infected with plague & 18 March 1545 \\
Claude Savoye & Woman dead of plague & 20 March 1545 \\
Michel Varro & House 'greased' & 20 March 1545 \\
Two debtors in prison & Dead of plague & 24 March 1545 \\
Ayme Revilliod & Infected house & 23 April 1545 \\
Jean Vollan (or Gentil) & Husband and children dead & 24 April 1545 \\
Bastien Bessonet & Possible plague death & 24 April 1545 \\
City prisons & Infected by plague & 24 April 1545 \\
Humbert Richard & Widow infected by plague & 27 April 1545 \\
Plague Hospital & forty infected & 11 May 1545 \\
Plague Hospital & Minister and barber infected & 11 May 1545 \\
Humbert Viennesin & Infected with plague & 21 May 1545 \\
Claude Choudens & Dead of plague & 10 November 1545 \\
Jean Ferron & Possible plague death & 26 July 1546 \\
Roz Porral & Wife infected & 27 September 1546 \\
Jean Magnin, labourer & Infected by plague & 15 October 1546 \\
\hline
\end{tabular}

